\title{
A Comprehensive Analysis of ACO for Wireless Sensor Network Routing
}

\author{
Nandini G, J Anitha, Keerthi Mohan
}

\begin{abstract}
The proliferations of IoT technologies and applications have led to an increased interest in Wireless Sensor Networks (and in particular, multi-hop networks). Wireless sensor networks are composed of small mobile terminals which have limited system resources. Due to this, these networks are vulnerable to changes in network status arising from changes in the network parameters such as, position / layout of sensors, signal strength, environmental conditions, etc. In addition, the network nodes are also constrained in terms of energy provided by the battery. It is an significant consideration to be accounted so as to prolong their operational time, since this adds to the network lifetime. Lot of research has gone into routing and transmission technologies for wireless sensor networks. Conventional routing mechanisms for WSNs still suffer from energy-hole problem caused by difficulties in adaptive route management. Thus, it is imperative that efficient routing mechanisms be developed in order to conserve energy and improve network lifetime. One popular approach is to use meta-heuristic algorithms for optimal path selection in a WSN route management system. A very popular meta-heuristic algorithm used for this objective is the Ant Colony Optimization (ACO) algorithms. ACO has been used as a base for many routing management systems. In this paper an extensive analysis of the performance of ACO based route selection mechanism is reported and also reporting a comparative analysis of efficacy of the ACO routing algorithm over the standard Greedy algorithm in finding routes with different count of sensor nodes and different count of ants. Then find that the ACO routing algorithm outdoes the Greedy algorithm with respect to the number of routes identified.
\end{abstract}

Keywords: ACO routing, Greedy algorithm, Meta-heuristic, Optimization, WSN..

\section{INTRODUCTION}

IoT (Internet of Things) is being touted as one of the next wave technological development of the century. In the IoT domain, Wireless Sensor Networks (WSNs) plays a vital part. The proliferation of WSNs has been brought about by recent advancement in sensor technology, wireless communication technology, intelligent computational algorithms, etc. WSNs are comprised of a number of sensor nodes distributed across

Revised Manuscript Received on August 15, 2020.

* Correspondence Author

Nandini G*, Research Scholor, VTU, AP/CSE, RRCE, Bangalore, Karnataka, India. Email:nanduamma@gmail.com

Dr. J Anitha, Professor/CSE, RVITM , Bangalore, Karnataka , India .Email: anithajayapalan@gmail.com.

Keerthi Mohan, AP/CSE, DSATM, Bengaluru, India. E-mail: keerthiimohan@gmail.com

(c) The Authors. Published by Blue Eyes Intelligence Engineering and Sciences Publication (BEIESP). This is an open access article under the CC BY-NC-ND license (http://creativecommons.org/licenses/by-nc-nd/4.0/) an area to be sensed / monitored. Modern sensors tend to be small, and are constrained in terms of computational and processing resources. Also, they are cheaper in comparison to conventional sensors. The data is acquired by the sensor nodes by sensing and measuring the environment. The data acquired is sent to the user depending on some deciding criteria. Modern sensor nodes are smart low energy devices having single or multiple sensors, a source of power, a small amount of memory, a processing unit, a radio for data transmission, and also an actuator. An assortment of magnetic, chemical, biological, optical, thermal, and mechanical sensors might be connected node for measuring the required attributes of the surrounding space / environment. As the sensor nodes are constrained by the amount of memory they have, and are usually installed in hard-to-access sites, a radio is employed for spatial broadcast of acquired information to a base station. Though the primary source of power in a sensor node is the battery, auxiliary power source, like solar panels can also be attached to the node depending upon the surrounding environment wherever the sensor is to be installed. Actuators might also be used with the sensors subject to the application.

Wireless Sensor Networks (WSNs) belongs to a kind of ad-hoc wireless networks wherein, the sensor nodes gather, process, and transmitt the data obtained from the surrounding location to an exterior Base Station (BS). As mentioned earlier, major bottlenecks in the development and enhancement of WSNs are: restricted power of the battery, small amount of memory, low computing power, and physical dimensions of the sensor nodes. Due to severe constraint in terms of energy of numerous densely deployed sensor nodes, we need to devise a set of network protocols to realize various network management and control functions such as network security, synchronization, etc. Routing is the procedure of selecting pathways among the sensor nodes within a WSN. The communication in a WSN involves node to node communication for transmission of the acquired data from the sensed space. This requires selection of the optimal route and route maintenance as well, so as to accomplish the application goals. Also, extending the lifetime of a WSN with restricted battery capacity is an important problem to be solved so as to boost the operating time of the sensor network as much as possible. Therefore, effective routing and communication technologies are crucial for achieving that goal.The routing protocol allows the network to choose a suitable path for the sensed data to be transmitted from the starting sensor node to the destination node. Routing procedure runs into quite a few difficulties while choosing the route depending upon, the network type, the radio channel properties,

Published By:

Blue Eyes Intelligence Engineering DOI: 10.35940/ijeat.F1628.089620

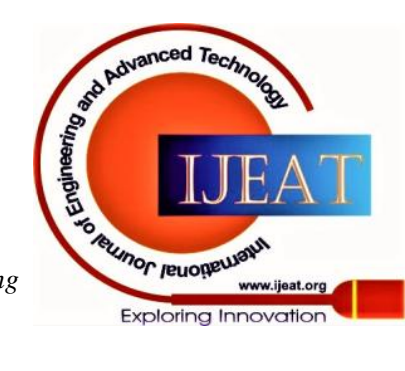




\section{A Comprehensive Analysis of ACO for Wireless Sensor Network Routing}

environmental conditions, any network layout changes, spontaneous node failures etc. In a small sensor network, the base station and sensor nodes are usually quite near such that they can directly communicate with one another. This type of communication is referred to as single-hop communication.

However, for many WSN applications, the area of coverage is so large that it may need hundreds to even thousands of nodes to be installed within the coverage zone. In such a situation multi-hop communication is desired as most of the sensor nodes are too distant from the destination node (or the gateway) due to which they cannot transfer data directly with the base station. In multi-hop communication network, the sensor nodes in addition to identifying the location and transmitting that information, also function as intermediate nodes for other nodes for transferring data with the base station. The onus of discovering an optimal path starting from the source to final node lies with the network layer. In this present work the focus is on identifying shortest communication path between the starting source node and the final destination node. Several protocols for routing have been proposed and implemented by various research groups. Some of these routing protocols are Direct Diffusion [1], LEACH [2], SPIN [2, 3]. Discovering a short way in between a source and destination is the bedrock of all routing techniques used in WSN. Thus, primarily it is a graph problem to invent the shortest path / route among two vertices of the graph. Conventional shortest path identification techniques are suitable for WSN due to resource constraints. In a multi-hop WSN, it needs to identify a path for data transmission such that it takes the least amount of time. This implies that the path should have least cost and minimal hop-count in order to accomplish this. Greedy algorithms like Dynamic Programming based algorithms, Bellman Ford algorithm, and Dijkstra's algorithm are useful in finding the shortest path. However, these algorithms tend to have high computational complexity. In addition, Greedy Algorithms do not guarantee globally optimal paths and hence are not suitable for dynamically changing network layout.

The traditional problem of finding the shortest path is an NP-hard problem is very computationally expensive. Maximization of network lifetime by optimizing network parameters in WSN routing process could be considered as a combinatorial optimization problem. In order to resolve the routing problem in WSN, alternate approaches using metaheuristic algorithms have been designed and proposed. Of late, the cooperative behavior of animals and insects such as ants, bats, bees, etc. have been studied in order to provide an analogous nature-inspired template to solve combinatorial optimization problem [4]. ACO algorithm simulate foraging behaviour of ants and is been effectively utilized in a number of optimization scenarios viz., vehicular traffic routing [5], asymmetric travelling salesman, and also network routing in WSN. In ACO, ants are the meta-heuristic agents which builds solutions during treading a traversal / while constructing graph. This cumulative quest behaviour renders ACO appropriate for determining combinatorial optimization problem's. Also, ACO exploits the domain knowledge (which is represented by the heuristic information) and search experiences (which is represented by pheromone) so as to speed-up the search process. In the present paper, it's been report an extensive evaluation of the basic ACO routing algorithm as applied to WSN routing problem. This involves studying the route optimization with respect to various parameters involved in the ACO algorithm. In Section - 2, a concise account of the existing work in the domain of routing for WSN using ACO is provided. In Section - 3, ACO routing technique is discussed in terms of the role of various parameters. Followed by that in Section -4 describes the results and discussion of the experimentation carried out. Finally, in Section - 5, includes conclusions regarding the performance of the ACO routing algorithm.

\section{LITERATURE SURVEY}

Taking into account the unpredictable nature of a WSN, an Adaptive ACO algorithm was introduced by $\mathrm{Ye}$ and Mohamadian [6] for cluster-based dynamic routing in a WSN. The implemented ACO algorithm finds the optimum network parameter setting for enhancing data aggregation leading to reduced data redundancy. Many ACO algorithms use ant memory to store the sequence of visited nodes. There are ACO approaches which are suitable for static nodes (Selcuk's method) [7], wherein, the data carried by an ant can be limited, leading to lower energy consumption. Here, the node's memory is used in order to save information related to other neighbouring nodes visited by an ant in addition to pheromone values. This gives the node the ability to accept or deny an incoming ant by looking up tabu list. Once an ant reaches the sink node, an acknowledgement message is returned to the node through the same path. Even though the method is very useful for searching best links in a large number of nodes, it has issues of prematurity and memory. This stems from the fact when a larger number of ants are used to simulate the network, it will lead to much higher traffic than the other methods. T Camilo et al. [8] proposed an improved ant colony routing named Energy Efficient Ant- Based Routing. They constructed a exclusive function to determine pheromone left by the remaining ants while returning back to their nest. The EEABR technique was designed to enhance the life of the sensor node by improving the energy usage at the node. This protocol makes use of pheromone tables in order to achieve energy efficient routing.

\section{ANT COLONY OPTIMIZATION AND WSN ROUTING USING ACO}

Marco Dorigo introduced the first ACO algorithms [9]. These algorithms were developed by studying the foraging behavior of ants. The inspiring factor was observing how ants build and discover shortest paths among the colony's nest and the source of food. Initially, as the ants start looking for food, they randomly survey the area surrounding their nest. Each ant places a trail of pheromone on the ground. Ants have senses which allow them to smell the pheromone. When subsequent ants leave the nest, they will be inclined to select, in probability, those paths which have stronger concentrations of the pheromone. Whenever an ant locates a origin of food, it assesses the quality and quantity of food. The ant then carries a small quantity of food back to the colony's nest. While coming back to the nest, the amount of chemical pheromone that an ant deposits along the pathway depends on the quality and of quantity the food discovered.

Published By:

Blue Eyes Intelligence Engineering and Sciences Publication (C) Copyright: All rights reserved.

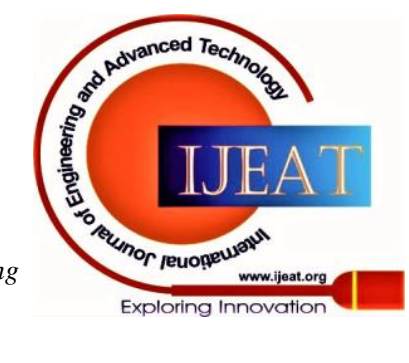


The following ants are driven by the pheromone trails to the source of food. This kind of indirect communication taking place amongst the ants by making use of the pheromone trails - referred to as stigmergy - aids them in identifying the optimal route between the colony's home to the food location.

In ACO based WSN routing, every ant attempts to find a network path such that it leads to minimal cost or effort. Ants which are dispatched from the source node s and navigate via the neighboring repeating nodes $y$, to finally arrive at the destination node $\mathrm{d}$. When data is available with a node which needs to be transported to the destination, further launching of ants is carried out. Once launched, choice of the subsequent node is performed based on the probabilistic decision making rule:

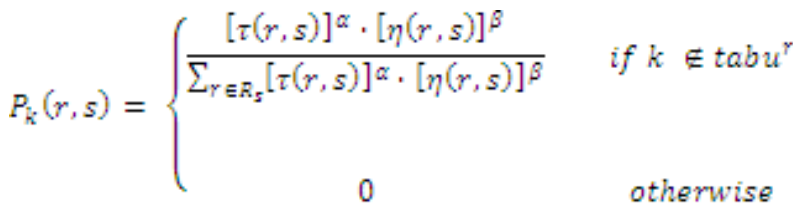

In this $\tau(\mathrm{r}, \mathrm{s})$ is equated to the pheromone level, $\eta(\mathrm{r}, \mathrm{s})$ is considered the heuristic value associated with energy, and $R_{s}$ the collection of receiver nodes. Also, a given node, is the listing of entries identifying the previously gathered data packets are factors / parameter's which regulate the relative influence of the pheromone trail and the heuristic value respectively. The pheromone trails are associated with the edges between sensor nodes. Each edge $(r, s)$ is associated to a trail value $\tau(\mathrm{r}, \mathrm{s}) . \in[0,1]$. As, destination node is fixed node, the final node in route remains constant for every ant traversal. Heuristic value associated with a node is stated as:

$$
\eta\left(r_{0} s\right)=\frac{\left(I-e_{y}\right)^{-1}}{\sum_{n \in R_{g}}\left(I-e_{n}\right)^{-1}}
$$

where the initial energy level is represented by I and the present energy level of the receiving node is represented by $r$. It permits making decision founded on the energy levels of the neighboring nodes. A node has lower probability to be selected in case it has a lesser energy level. All nodes notify their neighboring nodes regarding their levels of energy whenever any variation in their energy levels is detected.

The ACO algorithm also makes use of memory $\mathrm{M}_{\mathrm{k}}$ which the ant stores in its memory. This is done in order to keep track of the places / vertices visited by that ant. Since sequence numbers are used as the identity of each ant that previously visited a given node, these are stored within the node's memory, it is not necessary to keep $\mathrm{M}_{\mathrm{k}}$ lists within packet payload while transmission. As a result, this technique reduces the quantity of data to be transmitted, leading to saving of energy. In eqn (1) every receiver node has the option whether or not to receive the subsequent data packet related to ant, by examining its own tabu list. Hence, the receiving sensor node has the option whether or not to complete the receiving process by way of listening for the data packet and buffering it. In case the recipient node has previously received the same data packet, notification is sent to the transmitting node by sending out an ignore message, following which, it will switch over to idle mode while waiting for a new data packet to arrive.

Once all of the ants have finished their trip, every ant places a certain pheromone amount as shown by equation (3), wherein, , $\mathrm{Jw}^{\wedge} \mathrm{k}(\mathrm{t})$ is the length of the trip $\mathrm{Jw}^{\wedge} \mathrm{k}(\mathrm{t})$, is carried out by an ant $\mathrm{k}$ in an iteration $\mathrm{t}$. The quantity of the pheromone at every edge $1(r, s)$ of the nodes is expressed in eq (4). For WSNs, the count of nodes which are trvased by an ant $k$ on the trip $\mathrm{w}$ at an iteration $\mathrm{t}$ is represented by $\mathrm{Jw}^{\wedge} \mathrm{k}(\mathrm{t})$.

$$
\Delta \tau^{k}(t)=\frac{1}{j_{W}^{k}(t)}
$$

$\tau(\gamma, s)(t)+\tau(\gamma, s)(t)+\Delta \tau(r, s)(t), \forall V(\gamma, s) \in w^{k}(t), \quad k=1_{w m} m$

The pheromone levels are saved within the node's memory. Also, every node has knowledge of the pheromone level on the pathways to their neighboring nodes. At the end of every trip, a quantity of the pheromone trail $\Delta \tau^{\wedge} \mathrm{k}$ is deposited on the track treaded by the ant $\mathrm{k}$. This quantity of pheromone trail is constant for every edge $(r, s)$ traversed on a specific path. The update process is carried out by dispatching ant $\mathrm{k}$ returning to its own source node along the track, during the transmission of an acknowledge packet for the related data package. Enhancing pheromone levels on the pathways based on trip length, $J_{w}{ }^{k}(t)$, would continually set off a growing positive feedback. Hence, to restrain this build-up of pheromone level, a negative feedback mechanism involving evaporation of pheromone is applied at the culmination of every tour. This is shown in eq -5 wherein, the control parameter $\rho \in[0,1]$ is utilized in order to ascertain the degree of pheromone reduction through evaporation for every trip:

$$
\tau_{i, j}(t) \leftarrow(1-\rho) \tau_{i j p}(t)
$$

\section{RESULTS AND DISCUSSION}

In this Sec -4 , the conclusions of the computational experiments is presented. The wireless sensor network setup chosen was with 15 nodes in total with Node-1 and Node-2 being the source node and destination node, respectively. The coverage area considered is $1000 \mathrm{~m}$ by $1000 \mathrm{~m}$. The range of transmission for each sensor node is fixed at $500 \mathrm{~m}$. The source as well as destination nodes are fixed, while the remaining 13 nodes are placed randomly in the sensing space. With this setup, during a multi-hop transmission of a data packet from any node, only those nodes are considered which lie within the range of 500m from the node. Figure -1 depicts two different wireless sensor network layouts that were analyzed for ACO based routing. The free space attenuation factor has been set at 1.8. This attenuation factor generally ranges between 1.8 and 4.0. The number of ants is 10 with a pheromone level of 1.0 initially. The pheromone evaporation coefficient is set at 0.2 . In Figure -2 shows the normalized energy levels of sensors with respect to the iteration count for the two network layouts shown in Figure -1 .

The energy level of the sensor nodes keeps varying due to the fact that the route path keeps changing with iterations. However, the average energy consumption pattern is quite close to each other for both network layouts iteration of the ACO routing algorithm for evaporation rates.

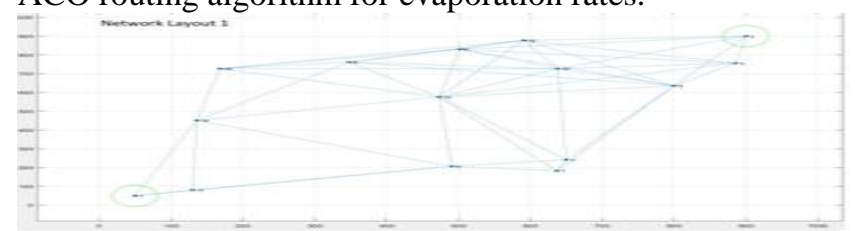

Published By:

Blue Eyes Intelligence Engineering and Sciences Publication (C) Copyright: All rights reserved.

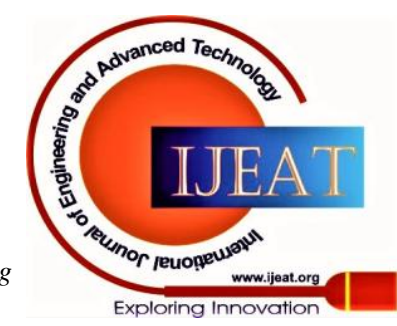




\section{A Comprehensive Analysis of ACO for Wireless Sensor Network Routing}

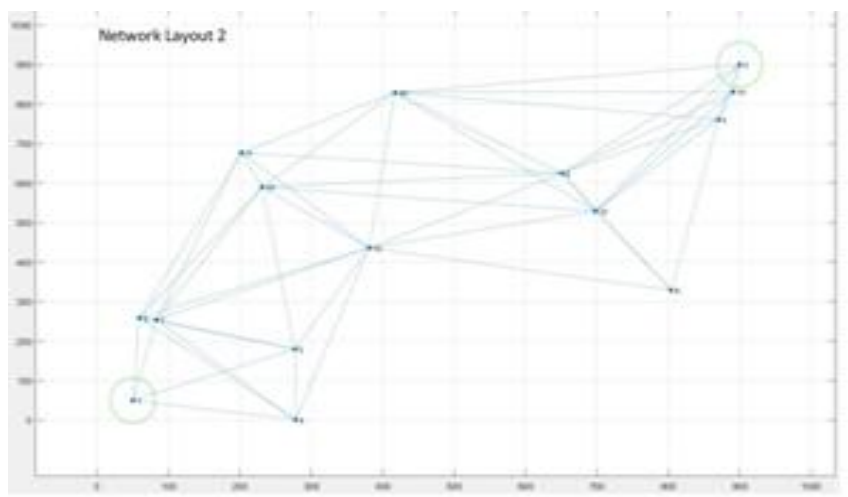

Figure - 1: Two wireless sensor network layouts considered for packet routing using the ant colony optimization.

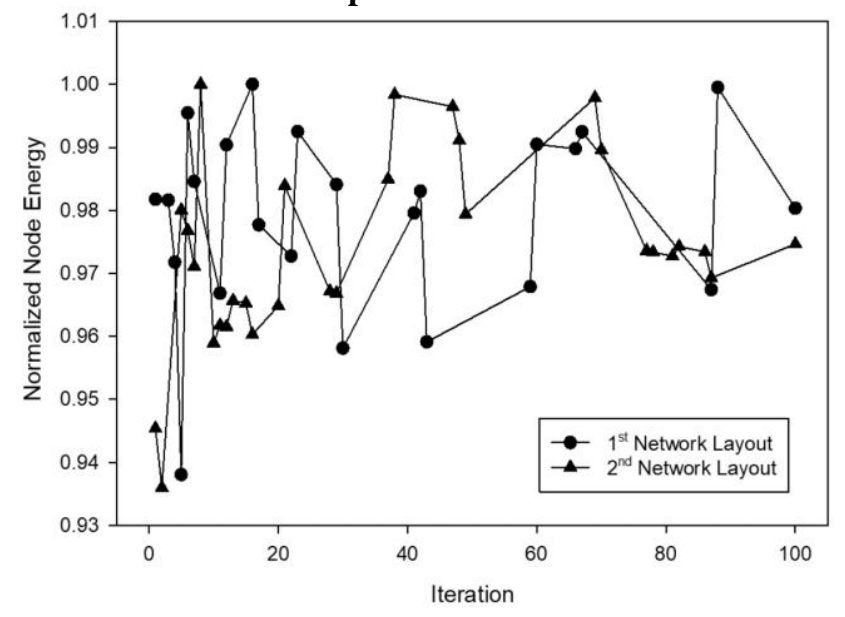

Figure - 2: Sensor node energy variation with iterations of the ACO routing algorithm for the two network layouts

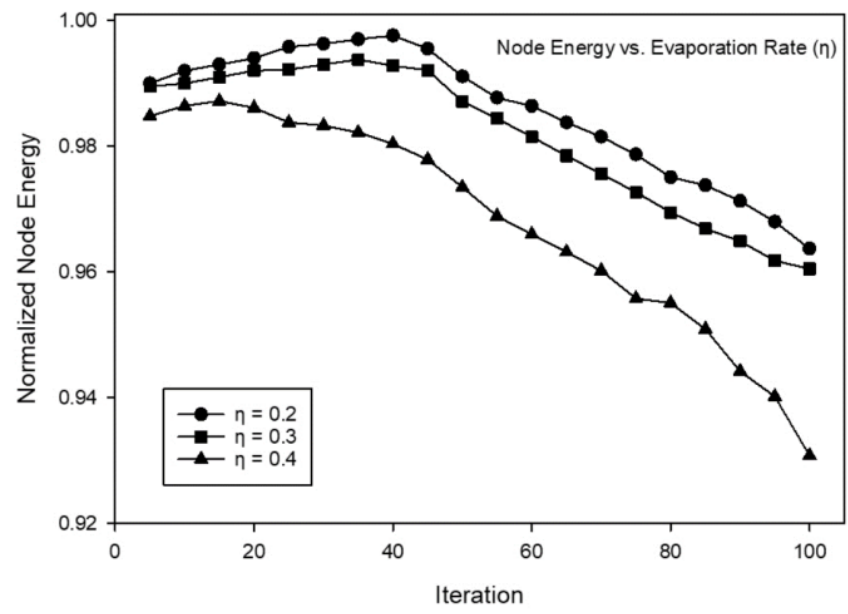

Figure - 3: Sensor node energy level with respect to iteration of the ACO routing algorithm for three different values of the pheromone evaporation rates.

In order to analyze the sensor node energy consumption model with respect to the pheromone evaporation rate, it's been fixed a 5-hop route identified by the ACO routing algorithm (out of many routes discovered by the algorithm). The energy level of the sensor nodes over 100 iterations for three values of pheromone evaporation values $(0.2,0.3$, and 0.4 ) is computed. The results of the computation are shown in Figure 3
Table - 1: Comparison of number of routes generated by Greedy Algorithm and the ACO Routing Algorithm with respect to number of sensor nodes and that of ants.

\begin{tabular}{|c|c|c|c|}
\hline \multirow{2}{*}{$\begin{array}{c}\text { No. of } \\
\text { Nodes }\end{array}$} & \multirow{2}{*}{$\begin{array}{c}\text { No. of } \\
\text { Ants }\end{array}$} & $\begin{array}{c}\text { Number of Routes } \\
\text { Algorithm }\end{array}$ & $\begin{array}{c}\text { ACO Routing } \\
\text { Algorithm }\end{array}$ \\
\hline 10 & 10 & 5 & 6 \\
\hline 10 & 15 & 3 & 4 \\
\hline 15 & 10 & 2 & 8 \\
\hline 15 & 15 & 6 & 14 \\
\hline 20 & 10 & 5 & 12 \\
\hline 20 & 15 & 10 & 21 \\
\hline
\end{tabular}

For further analysis of the efficiency of ACO routing algorithm, it has been performed numerical experiments for the number of routes identified and compared it with standard Greedy algorithm. In case of Greedy algorithm, first identified one route and then removed one node randomly from the path to discover more routes. This was carried out till the Greedy algorithm could no longer discover routes within the sensor network. Then for the same network layout, ACO routing algorithm was used to identify routes. For every run of the comparative analysis, a new random network layout was generated. It's been compared the number of routes generated by the two algorithms with 10, 15, and 20 sensor nodes and with 10 and 15 ants for each run. The comparison of the two algorithms is summarized in Table - 1, wherein, it is evident that ACO routing algorithm is able to identify a greater no of paths from source node to destination compared to the Greedy algorithm.

\section{CONCLUSIONS}

Route discovery using the standard Greedy Algorithm and compared with the ACO routing algorithm is performed. The number of viable routes identified by the ACO algorithm is more compared to the Greedy Algorithm. This indicates the superiority of the ACO routing algorithm over the Greedy algorithm. Analyzed a number of routes identified by the ACO routing algorithm and found that in most cases the sensor energy levels tend to decrease as the hop-count of the route increases. The pheromone evaporation rate is an indicator of the environmental conditions. A variation in pheromone evaporation level is analogous to changing radio profile of the space enclosing the WSN. As the pheromone evaporation rate increases, the sensor node energy level starts falling sharply with subsequent iterations. This shows that the network lifetime will get severely hampered with the rise in pheromone evaporation rate (environmental condition such as radio interference). ACO routing algorithm being a meta-heuristic, does indeed depend a lot on the computing power of the processor in the sensor node. With further development in the sensor electronics and better processors for use in sensor nodes, the performance of the ACO algorithm will certainly rise. Also, further enhancement and optimization of the ACO routing algorithm will enhance its applicability as a practical WSN routing solution power of the processor in the sensor node.

Published By:

Blue Eyes Intelligence Engineering and Sciences Publication

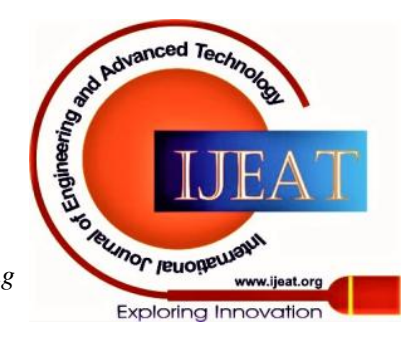


With further development in the sensor electronics and better processors for use in sensor nodes, the performance of the ACO algorithm will certainly rise. Also, further enhancement and optimization of the ACO routing algorithm will enhance its applicability as a practical WSN routing solution.

\section{REFERENCES}

1. "Synthetic structure of industrial plastics (Book style with paper title and editor)," in Plastics, G. O. Young, 2nd ed. vol. 3, J. Peters, Ed. New York: McGraw-Hill, 1964, pp. 15-64.

2. Directed Diffusion for Wireless Sensor Networking, Intanagonwiwat C., Govindan R., Estrin D., Heidemann J., and Silva F., IEEE/ACM Transactions on Networking, Vol. 11, No. 1, pp. 2 - 16, 2003.

3. Efficient Communication Protocol for Wireless Microsensor Networks, Heinzelman W.R., Chandrakasan A., and Balakrishnan H., Energy-Proceedings of the 33rdHawaii Intl. Conf. on System Sciences, pp. $1-10,2000$.

4. Negotiation-Based Protocols for Disseminating Information in Wireless Sensor Networks, Kulik J., Heinzelman W.R., and Balakrishnan H., Wireless Networks, Vol. 8, pp. 169 - 185, 2002

5. Swarm Intelligence. In Natural to Artificial Systems; Bonabeau E., Dorigo M., and Theraulaz G., Oxford Univ. Press: London, U.K., 1999.

6. Data Gathering Communication in Wireless Sensor Networks Using Ant Colony Optimization, Niannian D., Liu P.X., and Chao H., Proc. IEEE/RSJ International Conference on Intelligent Robots and Systems, pp. 697-702, 2005.

7. Adaptive Clustering based Dynamic Routing of Wireless Sensor Networks via Generalized Ant Colony Optimization, Ye Z., and Mohamadian H., IERI Procedia, Vol. 10, pp. 2 - 10, 2014.

8. Routing in wireless Sensor Network Using an Ant Colony Optimization (ACO) Router Chip, Okdem S., and Karaboga D., Sensors, pp. 909 921, 2009.

9. An Energy-Efficient Ant-Based Routing Algorithm for Wireless Sensor Networks, Proc. Intl. Camilo T., Carreto C., Sá Silva J., and Boavida F., Workshop on Ant Colony Optimization and Swarm Intelligence, Springer, Berlin, Heidelberg, pp.49 - 59, 2006.

\section{AUTHORS PROFILE}

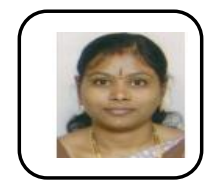

Nandini G, Is currently working as Assistant Professor in the Dept. Of Computer Science and Engineering at Rajarajeshwari College of Engineering, Bangalore, Karnataka, India. She received her Bachelor of Engineering (B.E), Master of Technology (M.Tech) from Visvesvaraya Technological University (VTU). Currently she is Pursuing Ph.D in VTU, Belagavi, Karnataka, India. Her research interests include Wireless Sensor Network, Internet of Things. Actively involved in professional organizations like CSI, ISTE..

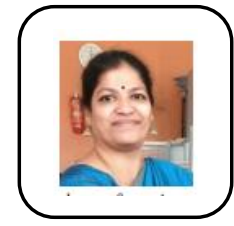

Dr J Anitha , is currently working as a Professor, Department of Computer Science and Engineering, DSATM, with an experience of 17 years. Graduated BE from KIT, Tiptur under Bangalore University in Computer Science and Engineering. She obtained her Master degree $\mathrm{ME}$ in Computer Science and Engineering from Anna University, Chennai and PhD degree from Anna University, Chennai in the area of Knowledge Based systems. She has 50 publications in International and National conferences, 20 publication in National journals and International Journals. Life Member of ISTE, CSI..

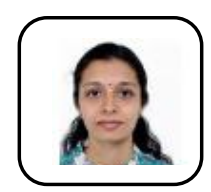

Keerthi Mohan: Is presently working as Assistant Professor in the department of Computer Science and Engineering at Dayananda Sagar Academy of Technology and Management, Bangalore, India. She received her Bachelor of Engineering(B.E) and Master of Engineering(M.E) in Computer Science and Engineering from Anna University, Chennai. She is pursuing her Ph.D in Vishvesaraya Technological University, Belagavi, Karnataka. Her research interests are big data analytics, Utility mining, Bio-inspired algorithms...

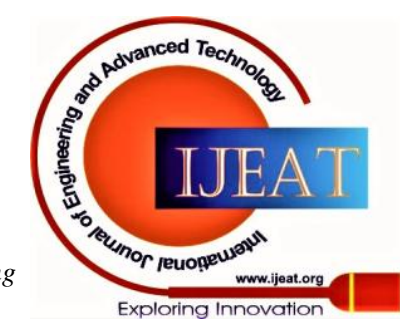

